\title{
Experimental investigation of collagen waviness and orientation in the arterial adventitia using confocal laser scanning microscopy
}

\author{
R. Rezakhaniha - A. Agianniotis - J. T. C. Schrauwen • \\ A. Griffa - D. Sage - C. V. C. Bouten • \\ F. N. van de Vosse • M. Unser • N. Stergiopulos
}

Received: 17 August 2010 / Accepted: 20 June 2011 / Published online: 10 July 2011

(C) The Author(s) 2011. This article is published with open access at Springerlink.com

\begin{abstract}
Mechanical properties of the adventitia are largely determined by the organization of collagen fibers. Measurements on the waviness and orientation of collagen, particularly at the zero-stress state, are necessary to relate the structural organization of collagen to the mechanical response of the adventitia. Using the fluorescence collagen marker CNA38-OG488 and confocal laser scanning microscopy, we imaged collagen fibers in the adventitia of rabbit common carotid arteries ex vivo. The arteries were cut open along their longitudinal axes to get the zero-stress state. We used semi-manual and automatic techniques to measure parameters related to the waviness and orientation of fibers.
\end{abstract}

R. Rezakhaniha and A. Agianniotis contributed equally to this work.

R. Rezakhaniha $\cdot$ A. Agianniotis $\cdot$ N. Stergiopulos

Laboratory of Hemodynamics and Cardiovascular Technology,

Institute of Bioengineering, EPFL, Lausanne, Switzerland

J. T. C. Schrauwen · F. N. van de Vosse

Cardiovascular Biomechanics, Department of Biomedical

Engineering, Eindhoven University of Technology, Eindhoven,

The Netherlands

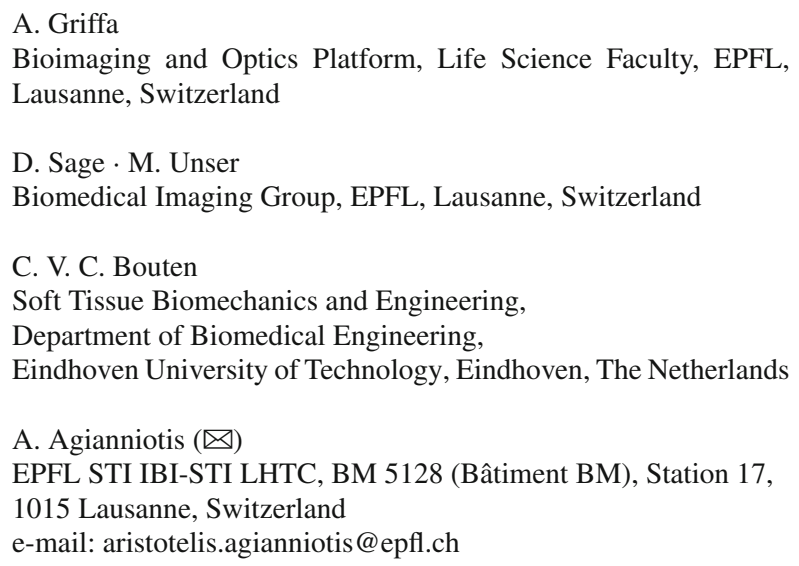

Our results showed that the straightness parameter (defined as the ratio between the distances of endpoints of a fiber to its length) was distributed with a beta distribution (mean value 0.72 , variance 0.028 ) and did not depend on the mean angle orientation of fibers. Local angular density distributions revealed four axially symmetric families of fibers with mean directions of $0^{\circ}, 90^{\circ}, 43^{\circ}$ and $-43^{\circ}$, with respect to the axial direction of the artery, and corresponding circular standard deviations of $40^{\circ}, 47^{\circ}, 37^{\circ}$ and $37^{\circ}$. The distribution of local orientations was shifted to the circumferential direction when measured in arteries at the zero-load state (intact), as compared to arteries at the zero-stress state (cutopen). Information on collagen fiber waviness and orientation, such as obtained in this study, could be used to develop structural models of the adventitia, providing better means for analyzing and understanding the mechanical properties of vascular wall.

Keywords Orientation distribution .

Waviness distribution - Collagen fibers - Arteries .

Common carotid artery

\section{Introduction}

Detailed biomechanical modeling of blood vessels should ideally be based on the structural properties of the main constituents of the wall with respect to its different layers. The adventitia is the outermost layer of blood vessels and its mechanical properties are determined mainly by the organization of collagen fibers in this layer. The two main features, characterizing collagen fiber organization, are its waviness and angular dispersion. Other factors that could determine the mechanical properties of the adventitia are collagen 
cross-linking, fiber density and glycoproteins (Williams et al. 2009; Et-Taouil et al. 2001).

Different techniques such as polarized light microscopy (PLM) (Hilbert et al. 1996; Canham et al. 1991; Smith et al. 1981; Finlay et al. 1995; Braga-Vilela et al. 2008; Rieppo et al. 2008; Whittaker and Canham 1991), small angle light scattering (SALS) (Ferdman and Yannas 1993; Sacks et al. 1997; Billiar and Sacks 1997), transmitted light microscopy (TLM) (Elbischger et al. 2005; Canham et al. 1989), fluorescence microscopy (FM) (Axer et al. 2001; Young et al. 1998), micro coherence tomography (Micro-CT) (Xia and Elder 2001), optical coherence tomography (OCT) (Hansen et al. 2002), small angle X-ray scattering (SAXS), second harmonic generation (SHG) (Boerboom et al. 2007; Wicker et al. 2008; Bayan et al. 2009) and electron microscopy (EM) (Franchi et al. 2007; Magnusson et al. 2002; Xia and Elder 2001; Wolinsky and Glagov 1964) have been used to characterize collagen bundle orientation and/or crimp properties in soft tissue. Fewer studies have focused on quantifying the organization of collagen fibers within the arterial wall (Canham et al. 1991; Smith et al. 1981; Finlay et al. 1995; Canham et al. 1989; Dingemans et al. 2000). The studies on arteries have been mainly conducted on pressurized (loaded) and/or chemically fixed and embedded vessels. These procedures could modify structural organization of tissue components (Dobrin 1996). Structural models of the mechanical response of the arterial wall are ideally based on the knowledge of the true zero-stress state (ZSS) of each wall constituent, preferably in wet fresh tissue where structural properties have not been changed. Krahn et al. have recently developed a new collagen specific fluorescence marker for tissues and live cell cultures (Krahn et al. 2006) which enables high resolution 3D imaging of collagen fibers in wet fresh tissues (Boerboom et al. 2007).

In this study, we have used the fluorescent marker developed by Krahn et al. combined with confocal microscopy and image analysis techniques to quantify the waviness and angular distribution of collagen fibers in the adventitia of fresh arteries at their zero-stress state. More specifically, this study was designed to (a) quantify the waviness properties of collagen fibers, (b) determine how the waviness distribution of fibers depends on their main orientation and (c) measure the local angular distribution of collagen fibers in the adventitia of rabbit common carotid arteries.

\section{Materials and methods}

\subsection{Tissue preparation}

Fourteen common carotid arteries were excised postmortem from seven New Zealand white male rabbits weighing $3 \pm$ $0.1 \mathrm{~kg}$ from a local slaughterhouse. We selected to use com- mon carotid arteries of rabbits because they exhibit little tapering and their properties are fairly uniform along their length. Arteries were transported on ice in phosphate buffered saline (PBS) to the laboratory and cleaned from the surrounding tissue. To visualize collagen fiber, the arteries were incubated over night at $37^{\circ}$ in a $2 \mu \mathrm{M}$ solution of CNA35-OG488 in PBS, which was used as the specific vital florescent marker for vascular collagen (Krahn et al. 2006). After incubation, the common carotid artery was cut into two equal segments of approximately $25-30 \mathrm{~mm}$ in length. One segment was used to measure collagen waviness and orientation in the zero-stress state, which was achieved by cutting open carefully along the artery's axis (cut-open group) and then immersed in PBS for 30 min before imaging (Han and Fung 1996). The other group was kept intact (intact group) at its zero-load state. All measurements were done within $24 \mathrm{~h}$ of excision.

\subsection{Confocal microscopy}

We used an upright confocal microscope (Leica SP5 white laser, Leica, Germany) to visualize collagen fibers labeled with the fluorescent marker CNA35-OG488. Arteries were secured by a pin on a silicone coated Petri dish filled with PBS, with the adventitia facing upwards (Fig. 1). The thickness of the adventitia layer of rabbit carotid arteries is about $0.13 \mathrm{~mm}$. Two circumferential lines close to each border were marked by a blue Miller's elastic stain as shown in Fig. 1 . The distance between these lines was measured before and after fixing the tissue on the Petri dish to ensure that the arteries were not stretched. In addition, the field of view was rotated in a way that the horizontal axis of images was parallel with the axial axis of the vessel. The fluorescent marker was excited at $488 \mathrm{~nm}$ by an Argon laser and a photomultiplier tube (PMT) detector was defined at $498-550 \mathrm{~nm}$ to collect the emitted fluorescent signal. Ten arterial segments per group were scanned at 5 different locations along the longitudinal axis. At each location, a $z$-stack with a step size of $0.5 \mu \mathrm{m}$ was obtained, resulting in a total image size of $238 \mu \mathrm{m} \times 238 \mu \mathrm{m} \times \sim 100 \mu \mathrm{m}$, with $512 \times 512$ pixels in each 2D image. In total, $50 z$-stacks were imaged for each group. Figure 1 shows an example of the image stack after 3D reconstruction by IMARIS software (Bitplane, Switzerland).

\subsection{Image analysis}

\subsubsection{D measurement of fiber angle}

The orientation of collagen bundles in two representative $z$-stacks from each group was measured by manually locating endpoints of each collagen bundle using IMARIS Software. Figure 2 shows an example of the 3D reconstruction of collagen fibers from the $z$-stacks. Fibers have been manually 
Fig. 1 The arterial segment is placed on a silicon-coated Petri dish (not shown) with the adventitia facing upwards and an example of the $3 \mathrm{D}$ reconstruction of outermost layer of adventitia is presented. $\theta$ is the circumferential direction and $z$ the axial direction

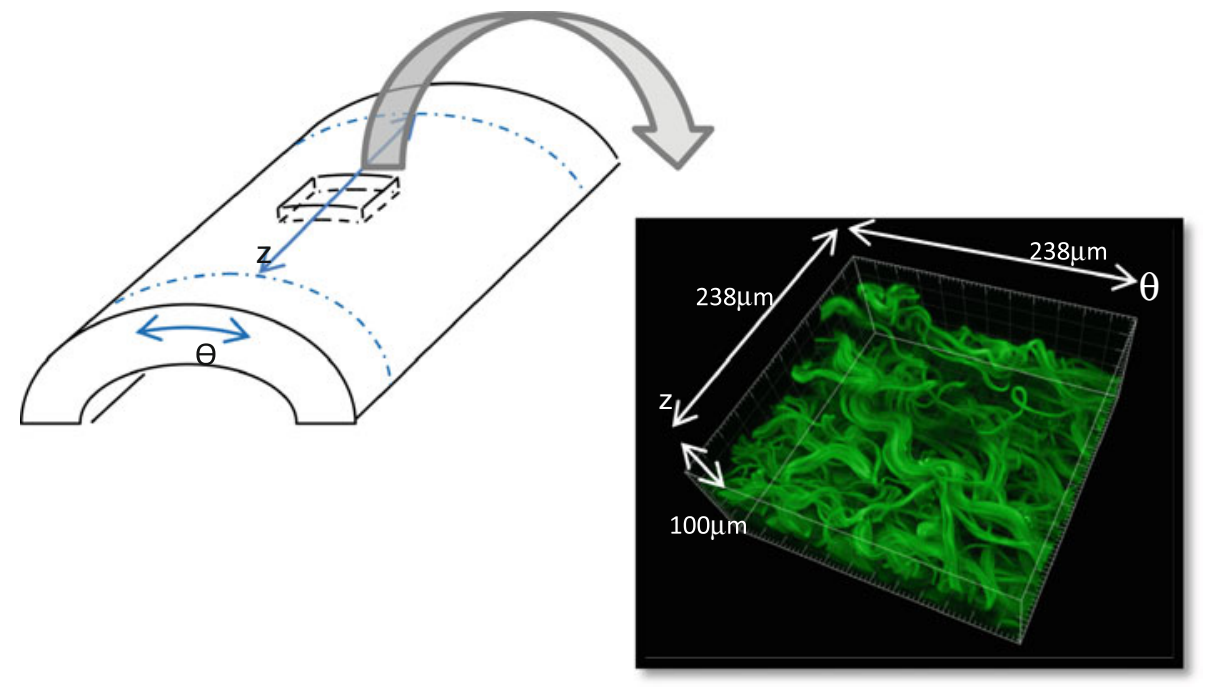

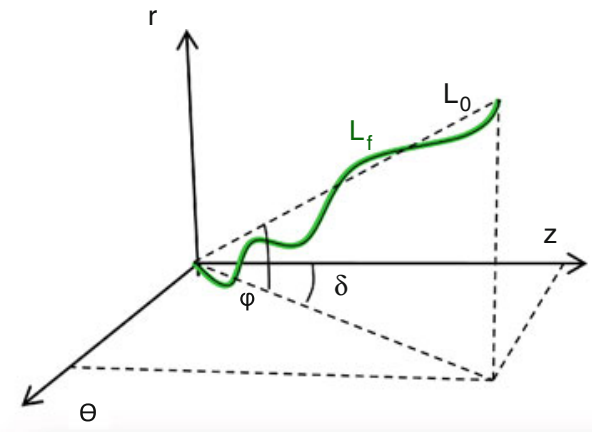

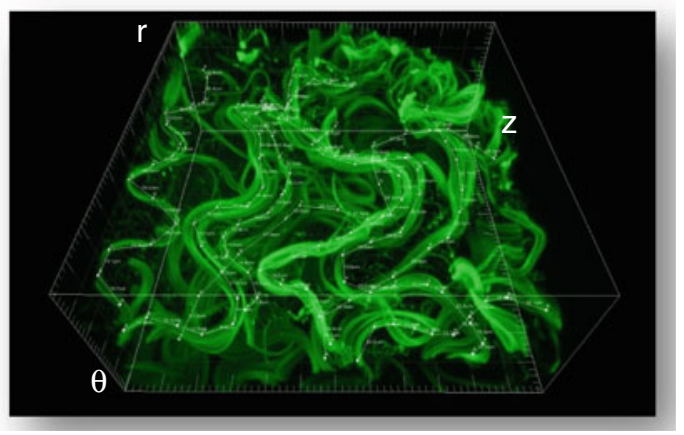

Fig. 2 Latitude (radial) angle $\varphi$ measured by manual tracing of fibers in a representative $3 \mathrm{D}$ reconstruction of $z$-stacks. $z, \theta, r$ correspond to the longitudinal, circumferential, and radial directions

traced and the global orientation of each fiber has been measured in a spherical coordinate system. The orientation of a fiber in 3D could be therefore described by the azimuthal angle $\delta$ (between $-\pi / 2$ and $\pi / 2$ ) and the latitude (radial) angle $\varphi$ (between 0 and $\pi$ ) as shown schematically in Fig. 2 .

\subsection{2 $2 \mathrm{D}$ image analysis}

Based on the assumption that the radial angle $\varphi$ is small and in order to facilitate the image analysis, the $z$-stacks

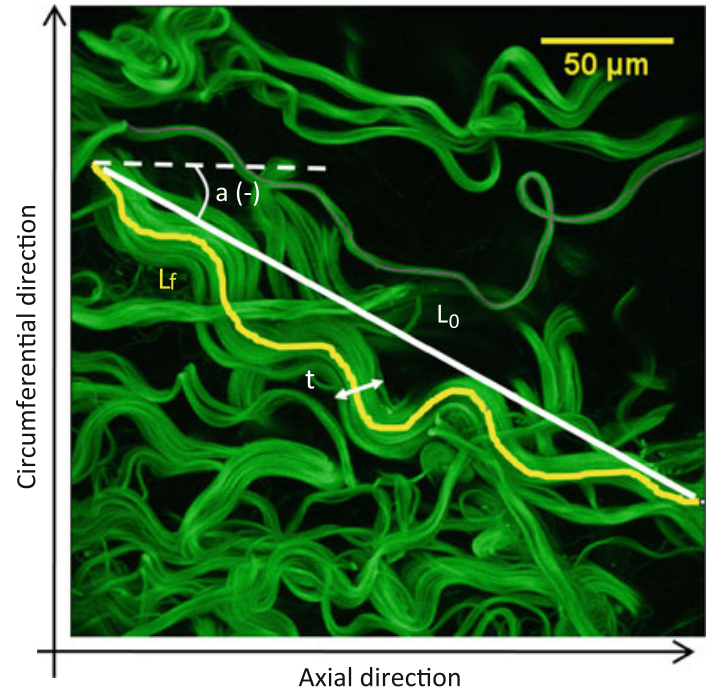

Fig. 3 Definition of measured parameters i.e., global angle $(a)$, thickness $(t)$, and length $\left(L_{f}\right)$ of a fiber bundle as well as the length $\left(L_{o}\right)$ of a straight line connecting the ends of the measured fiber bundle

were flattened using the maximum intensity projection (MIP) available in ImageJ software (NIH, Bestheda, Maryland, USA).

2.4 Measurement of waviness and global angle of collagen bundles

To quantify the waviness characteristics and global angle of collagen bundles in the zero-stress state, all 2D images from the cut-open group were analyzed semi-automatically. Figure 3 shows the parameters measured for each collagen bundle on the image i.e., the distance between visible endpoints of a collagen bundle $\left(\mathrm{L}_{0}\right)$, the length of the fiber bundle $\left(\mathrm{L}_{f}\right)$, the thickness $(t)$ measured in three different locations along each bundle, and the global angle of bundles (a) which 
is the angle between the main axis of the fiber and the axial direction $z$ as specified in Fig. 3. This angle could take values between -90 and 90 degrees. These parameters were measured using NeuronJ, an ImageJ plug-in originally developed for Neurite tracing and analysis (Meijering et al. 2004). The plug-in facilitates following the fibers on a $2 \mathrm{D}$ image and gives as the output the fiber length $\left(\mathrm{L}_{f}\right)$ as well as the coordinates of the endpoints. Most bundles are larger than the field of view. Therefore, we emphasize that these parameters are measured for the part of the bundle visible in the field of view.

To quantify the waviness of fibers, we introduced the straightness parameter $\left(P_{S}\right)$ defined as,

$P_{s}=L_{0} / L_{f}$

$P_{S}$ is bounded between 0 and 1 . The lower the value of $P_{s}$, the wavier the fiber is. A bundle with $P_{s}=1$ indicates a totally straight fiber. $P_{s}$ converges to zero when the fibers get very wavy. This parameter could be particularly useful for modeling the gradual engagement of wavy collagen fibers which will be discussed later. In addition, we estimated the size of the bundle by,

$\tilde{S}=L_{f} \cdot \bar{t}$

where $\bar{t}$ is the mean thickness of collagen bundles. Thus, each bundle could be characterized by three parameters, i.e., a straightness parameter $P_{S}$, a global angle and an estimated size $\tilde{S}$. To give more importance to measurements from larger bundles which contain more collagen fibers than the smaller ones, in the estimation of mean straightness parameter $P_{S}$ and the mean angle a, the individual bundle values of $P_{S}$ and a were weighted by the size $\tilde{S}$ of the corresponding bundle.

\subsection{Waviness distribution analysis}

To quantify the waviness characteristics of collagen fibers, we analyzed the distribution of the straightness parameter. First, we used a bi-variate histogram to visualize the frequency of straightness parameter $P_{S}$ and the global angle a, based on the weighted dataset. Second, the dataset was used to plot a univariate probability density for the straightness parameter regardless of the global angle using MATLAB (The MathWorks, USA). This means that the probability density was extracted from all values of $P_{S}$ in the dataset. A beta and an extreme value distribution were fitted to the $P_{S}$ values. Beta probability distribution is a statistical distribution defined between 0 and 1 as,

$f\left(P_{s} ; \alpha, \beta\right)=\frac{P_{s}^{\alpha-1}\left(1-P_{s}\right)^{\beta-1}}{B(\alpha, \beta)}, \quad 0<P_{s}<1$ where $\alpha$ and $\beta$ are shape parameters of the distribution and $\mathrm{B}(\alpha, \beta)$ is the beta function,

$B(\alpha, \beta)=\int_{0}^{1} t^{\alpha-1}(1-t)^{\beta-1} \mathrm{~d} t$

As for the extreme value distribution, it is defined as,

$f\left(P_{s} ; \mu, \sigma\right)=\sigma^{-1} \exp \left(\frac{P_{s}-\mu}{\sigma}\right) \exp \left(-\exp \left(\frac{P_{s}-\mu}{\sigma}\right)\right)$

where $\mu$ is the location parameter and $\sigma>0$ the scale parameter.

Third, to decide whether $P_{S}$ is distributed more accurately by a beta or an extreme value distribution, we have used probability plots of the data for both distributions using statistics toolbox $^{\mathrm{TM}}$ available in MATLAB. The probability plot is a graphical technique for assessing whether or not a dataset follows closely a given distribution. The plot includes a reference line useful for judging whether the data follow well or not the particular distribution. The closer the probability plot of data to this line, the better the data follow that particular distribution (Chambers et al. 1983). Finally, using the bivariate histogram, the weighted data were divided to six groups based on their global angle a, starting from -90 with $30^{\circ}$ steps, and the probability density of the straightness parameter for each angle group was determined. Then, we fitted the beta distribution to all six groups of fibers and compared the distribution parameters. This step helped us to decide whether the distribution of the straightness parameter $P_{s}$ depends on the global angle or not.

\subsection{Local angle analysis}

\subsubsection{Image analysis tool}

To estimate the local orientation of the collagen fibers, we used OrientationJ, which is an ImageJ plug-in developed inhouse based on structure tensors. Structure tensors are matrix representatives of partial derivatives and are commonly used in the field of image processing (Jahne 1993; Bigun et al. 2004). OrientationJ evaluates the local orientation and isotropic properties (coherency and energy) of every pixel of the image. These values are derived from the structure tensor defined for each pixel as the $2 \times 2$ symmetric positive matrix $J$,

$J=\left[\begin{array}{c}<f_{x}, f_{x}>_{w}<f_{x}, f_{y}>_{w} \\ <f_{x}, f_{y}>_{w}<f_{y}, f_{y}>_{w}\end{array}\right]$

where $f_{x}$ and $f_{y}$ are the partial spatial derivatives of the image $f(x, y)$, along the principal directions $\mathrm{x}$ and $\mathrm{y}$, respectively. Furthermore, the weighted inner product between two 
arbitrary images $\mathrm{g}$ and $\mathrm{h}$ is defined as,

$$
<g, h>_{w}=\iint_{R^{2}} w(x, y) g(x, y) h(x, y) \mathrm{d} x \mathrm{~d} y
$$

$w(x, y)$ is the Gaussian weighting function that specifies the area of interest. Once the structure tensor is known, local orientation, energy and coherency for each pixel could be easily calculated (Jahne 1993). The local predominant orientation $\theta$ in the considered region corresponds to the direction of the largest eigenvector of the tensor and it is thus given by,

$\theta=\frac{1}{2} \arctan \left(2 \frac{<f_{x}, f_{y}>_{w}}{<f_{y}, f_{y}>_{w}-<f_{x}, f_{x}>_{w}}\right)$

The energy parameter $E$ is the trace of the tensor matrix,

$E=\operatorname{Trace}(J)=<f_{x}, f_{x}>_{w}+<f_{y}, f_{y}>_{w}$

Pixels with higher energy values correspond to less isotropic and more clearly oriented structures.

The coherency parameter $C$ is defined as the ratio between the difference and the sum of the tensor eigenvalues,

$$
\begin{aligned}
C & =\frac{\lambda_{\max }-\lambda_{\min }}{\lambda_{\max }+\lambda_{\min }} \\
& =\frac{\sqrt{\left(<f_{y}, f_{y}>_{w}-<f_{x}, f_{x}>_{w}\right)^{2}+4<f_{x}, f_{y}>_{w}}}{<f_{x}, f_{x}>_{w}+<f_{y}, f_{y}>_{w}}
\end{aligned}
$$

where $\lambda_{\max }$ is the largest and $\lambda_{\min }$ the smallest eigenvalue, respectively. Coherency is bounded between 0 and 1 , with 1 indicating highly oriented structures and 0 indicating isotropic areas.

In OrientationJ, the tensor $J$ is evaluated for each pixel of an input image by computing the continuous spatial derivatives in $x$ and $y$ using a cubic B-spline interpolation (Unser et al. 1993). The local orientation, coherency, and energy are computed based on Eqs. 8, 9 and 10. In our directional analysis, we included only the angular values from parts (pixels) of the images which effectively correspond to fiber edges, as the orientation information from uniform areas is not significant. To do this, we used the energy of derivatives and the coherency values to discriminate between significantly and not-significantly oriented areas. Therefore, the histogram of the orientation is built with pixels which have at least $2 \%$ of normalized energy. Moreover, the orientation values in the histograms are weighted by the coherency values to give more importance to the orientations which correspond to elongated structures in the local neighborhood.

We made the OrientationJ plug-in and an ImageJ macro for angle analysis batching available online at http://bigwww. epfl.ch/demo/orientation. OrientationJ computes the orientation, energy and coherency maps of an image and its weighted orientation histogram. It is possible to set thresholds on the energy and coherency maps to select the image orientation values to be included in the angles histogram. The plug-in also outputs a hue-saturation-brightness (HSB) color-coded map which shows the angles of the oriented structures in the image as shown in Fig. 4.

\subsubsection{Fitting local angles}

Using OrientationJ plug-in for ImageJ, we obtained the histogram of local angles for all individual images of collagen fibers. Next, we summed up the individual histograms from images in each group to calculate local orientation of both cut-open and intact groups. The probability density function (PDF) of the local angles for each group was then calculated by normalizing local angular histograms. We fitted an empirical PDF by a four modal directional PDF representing 4 families of fibers. The angular distribution of each family was modeled with a $\pi$-periodic von Mises distribution (Mardia and Jupp 2000) which is the $\pi$-periodic equivalent of a Gaussian distribution in directional statistics,

$R(\theta ; \phi, b)=\frac{1}{\pi I_{0}(b)} e^{b \cos (2(\theta-\phi))}-\frac{\pi}{2} \leq \theta<\frac{\pi}{2}$

$I_{0}$ denotes the modified Bessel function of the first kind and order 0 , which is defined as,

$I_{0}(b)=\frac{1}{2 \pi} \int_{0}^{2 \pi} e^{b \cos \theta} \mathrm{d} \theta$

The parameter $\varphi$ is the mean orientation and the parameter $b$ is the concentration parameter.

The distribution is symmetric about $\theta=\varphi$ and the $\operatorname{circu}$ lar standard deviation $\sigma$ of this distribution is (Mardia and Jupp 2000),

$\sigma=\left\{-2 \log \left(\frac{I_{1}(b)}{I_{0}(\beta)}\right)\right\}^{1 / 2}$

$I_{1}$ denotes the modified Bessel function of the first kind and order 1 ,

$I_{1}(b)=\frac{1}{2 \pi} \int_{0}^{2 \pi} \cos \theta e^{b \cos \theta} \mathrm{d} \theta$

We assumed cylindrical symmetry around the $z$ axis of arteries. Based on this assumption and the shape of the empirical PDF, families of fibers to fit the data were assumed having mean angles of $\varphi_{1},-\varphi_{1}, \pi / 2$ and 0 with shape parameters 


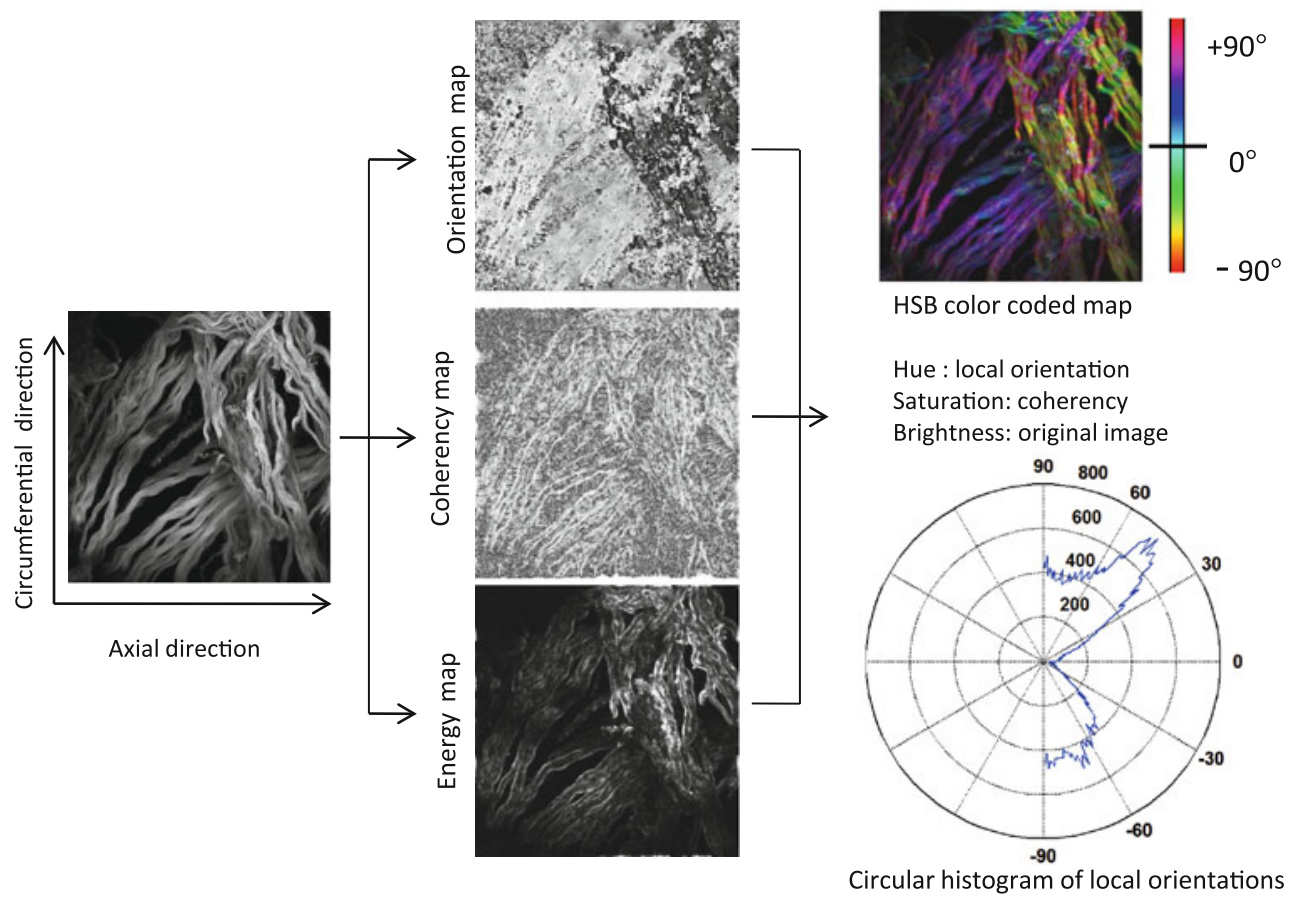

Fig. 4 Results of the OrientationJ, the imageJ plug-in developed to get the histogram of local angles, the orientation, energy and coherency maps of an image and the HSB color-coded map

$b_{1}, b_{2}, b_{3}$ and $b_{4}$, respectively. Therefore the fitted probability density distribution was defined as,

$$
\begin{aligned}
T R & \left(\theta ; \varphi_{1}, b_{1}, b_{2}, b_{3}, b_{4}\right) \\
= & \frac{1}{4} R\left(\theta ; \varphi_{1}, b_{1}\right)+\frac{1}{4} R\left(\theta ;-\varphi_{1}, b_{2}\right) \\
& +\frac{1}{4} R\left(\theta ; \frac{\pi}{2}, b_{3}\right)+\frac{1}{4} R\left(\theta ; 0, b_{4}\right)-\frac{\pi}{2} \leq \theta<\frac{\pi}{2}
\end{aligned}
$$

\subsection{Transformation law}

The deformation of the arterial wall is assumed as an affine deformation. Furthermore, in order to simplify the analysis, the collagen bundles are assumed to lie only in the circumferential-axial plane. Based on the above assumptions, one can obtain the density probability of local orientations in the deformed state (intact) based on the zero-stress state (cut-open). As seen in Fig. 5, if $\lambda_{\theta}$ and $\lambda_{z}$ are the stretches in the circumferential and axial directions, respectively, and $\theta_{0}$ and $\theta_{1}$ are the local angles of fibers with respect to the axial direction in the initial state (cut-open) and the deformed state (intact), respectively; the angles are then related to each other by,

$\tan \theta_{1}=\frac{\lambda_{\theta}}{\lambda_{z}} \tan \theta_{0}$

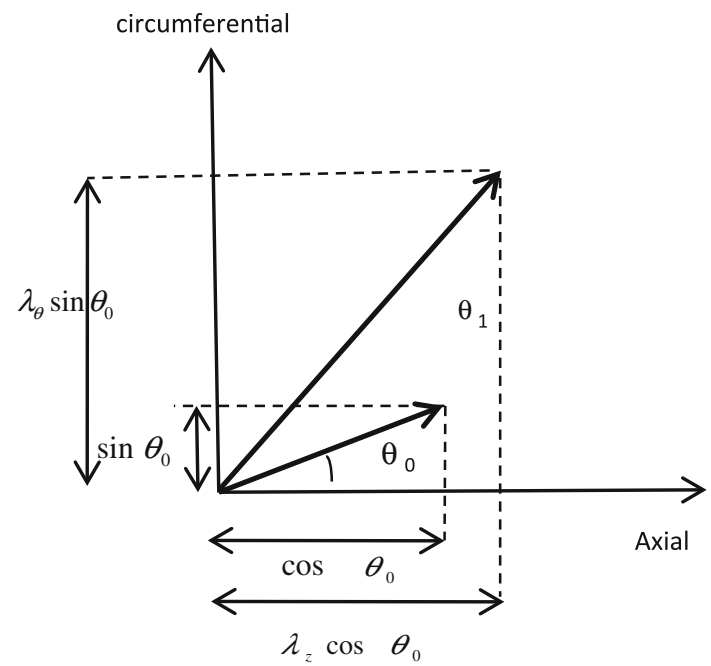

Fig. 5 Mapping of local angles from the original state $\left(\theta_{0}\right)$ to the deformed state $\left(\theta_{1}\right)$. The angles $\theta_{0}$ and $\theta_{1}$ are related to each other with Eq. 16. $\lambda_{\theta}$ and $\lambda_{z}$ are the stretches in the circumferential and axial directions, respectively

$R_{0}$ and $R_{1}$ are density distributions for local angles between $-\pi / 2$ and $\pi / 2$. Therefore,

$\int_{-\pi / 2}^{\pi / 2} R_{0}\left(\theta_{0}\right) \mathrm{d} \theta_{0}=\int_{-\pi / 2}^{\pi / 2} R_{1}\left(\theta_{1}\right) \mathrm{d} \theta_{1}=1$ 
The function of $\theta_{1}$ versus $\theta_{0}$ is monotonic and the above equation results in,

$$
\begin{aligned}
& R_{0}\left(\theta_{0}\right) \mathrm{d} \theta_{0}=R_{1}\left(\theta_{1}\right) \mathrm{d} \theta_{1} \\
& R_{1}\left(\theta_{1}\right)=R_{0}\left(\theta_{0}\right) \frac{\mathrm{d} \theta_{0}}{\mathrm{~d} \theta_{1}} \\
& =R_{0}\left(\theta_{0}\right) \frac{\cos ^{2} \theta_{0}}{\frac{\lambda_{\theta}}{\lambda_{z}} \cos ^{2}\left(\tan ^{-1}\left(\frac{\lambda_{\theta}}{\lambda_{z}} \tan \theta_{0}\right)\right)}
\end{aligned}
$$

Therefore, if $R_{0}$ is known, $R_{1}$ could be calculated based on the ratio of $\lambda_{\theta} / \lambda_{z}$ and the distribution $R_{0}$. The transformation law has been applied on the data to find out the corresponding ratio of $\lambda_{\theta} / \lambda_{z}$.

\section{Results}

In this study, we have used manual, semi-automatic, and automatic techniques to quantify the statistical distribution of parameters related to the orientation and waviness of fibers in the adventitia of rabbit carotid arteries.

Figure 2 shows an example of the 3D reconstruction of collagen fibers from the $z$-stacks. The latitude (radial) angles, manually measured from the 3D reconstruction of samples, are listed in Table 1 . The mean value of the absolute radial angle was measured as $5.6^{\circ}$ and $6.1^{\circ}$ in the cut group and $4.8^{\circ}$ and $1.7^{\circ}$ in the uncut group. Based on these small angle values, the fibers were practically lying in the $z-\theta$ (axialcircumferential) plane in both groups. This trend was also observed visually in all $z$-stacks.

Figure 6 shows the weighted frequency of bivariate data, i.e., the set of global orientations and straightness from the cut group. The same dataset was used to create Figs. 7 and 9. Figure 7 shows the probability density of the straightness parameter $P_{s}$ regardless of the global angle of the data. This

\begin{tabular}{|c|c|c|c|c|}
\hline \multirow{2}{*}{$\begin{array}{l}\text { Group } \\
\text { Sample }\end{array}$} & \multicolumn{2}{|l|}{ Cut } & \multicolumn{2}{|c|}{ Uncut } \\
\hline & S1 & $\mathrm{S} 2$ & S3 & $\mathrm{S} 4$ \\
\hline \multirow[t]{8}{*}{$\varphi(\operatorname{deg})$} & 13.8 & 3.0 & 5.4 & -1.2 \\
\hline & 9.2 & 3.0 & 4.6 & -2.7 \\
\hline & 3.3 & 7.0 & 3.8 & 0.3 \\
\hline & -9.0 & 14.9 & -4.6 & -0.8 \\
\hline & 0.0 & 6.1 & -2.8 & 2.0 \\
\hline & -0.6 & 9.6 & -8.0 & 4.6 \\
\hline & -3.4 & 3.2 & -3.3 & 1.5 \\
\hline & -5.9 & 2.3 & -5.6 & 0.7 \\
\hline Mean $(|\varphi|)$ & 5.6 & 6.1 & 4.8 & 1.7 \\
\hline std $(|\varphi|)$ & 4.7 & 4.3 & 1.6 & 1.4 \\
\hline
\end{tabular}

Table 1 Latitude (radial) angle measurements of 4 representative samples

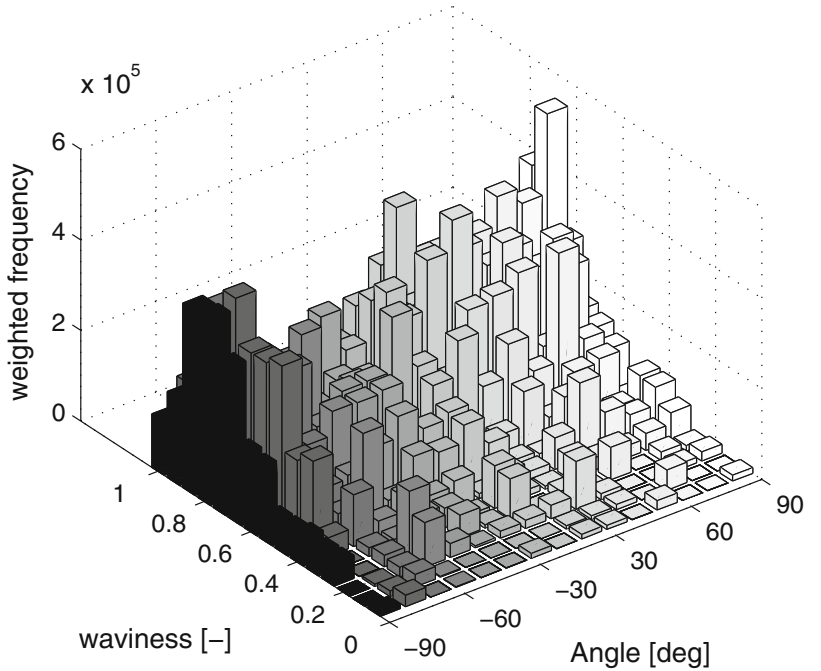

Fig. 6 Bivariate histogram of straightness parameter $\left(\mathrm{P}_{s}\right)$ and global angles with respect to the axial direction of the artery based on the entire weighted dataset

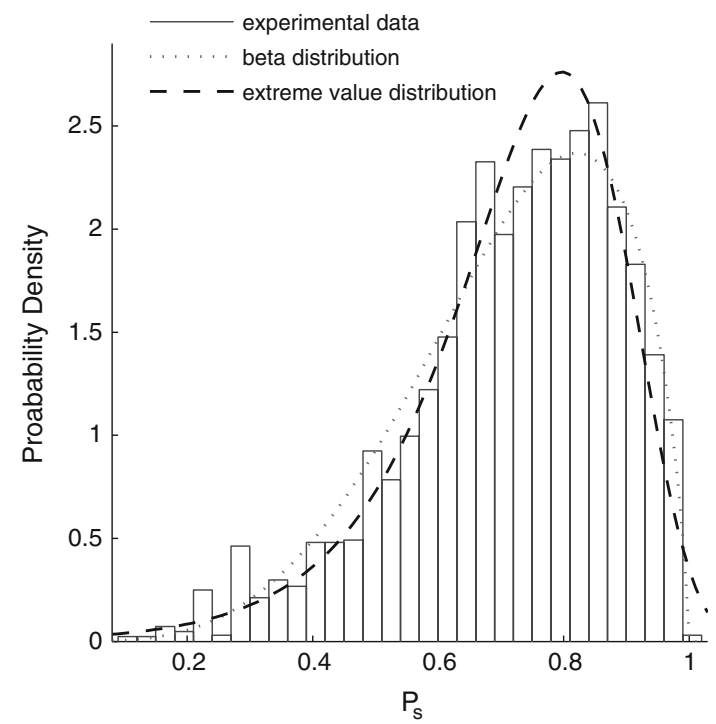

Fig. 7 Univariate probability density of straightness parameter $\left(\mathrm{P}_{S}\right)$ using all weighted data regardless of the global angle: empirical and corresponding fits from beta and extreme value distributions

means simply that Fig. 7 plots the density of all weighted straightness parameters $P_{S}$. In addition, Fig. 7 includes the best fits for beta and extreme value distributions on the experimental density distribution of the straightness. Parameter values from best fits are reported in Table 2 . The best fit for the beta distribution resulted in $\alpha=4.47$ and $\beta=1.76$ and the best fit for the extreme value distribution resulted in $\mu=0.800$ and $\sigma=0.133$. To assess how well the dataset followed each of these distributions, the probability plots of the data for both distributions were plotted in Fig. 8. Based on Fig. 8, the data are closer to the straight line for the beta distribution than the extreme value distribution. This indicates 
Table 2 Parameters of the distribution fits on straightness parameter, Ps

\begin{tabular}{llll}
\hline Distribution & Extreme values & Distribution & Beta \\
\hline Log likelihood & 2377 & Log likelihood & 2480 \\
Domain & $-\infty<y<\infty$ & Domain & $0<y<1$ \\
Mean & 0.720 & Mean & 0.718 \\
Variance & 0.029 & Variance & 0.028 \\
Parameter estimate & & Parameter estimate \\
$\mu$ & 0.800 & $\alpha$ & 4.47 \\
$\sigma$ & 0.133 & $\beta$ & 1.76 \\
\hline
\end{tabular}
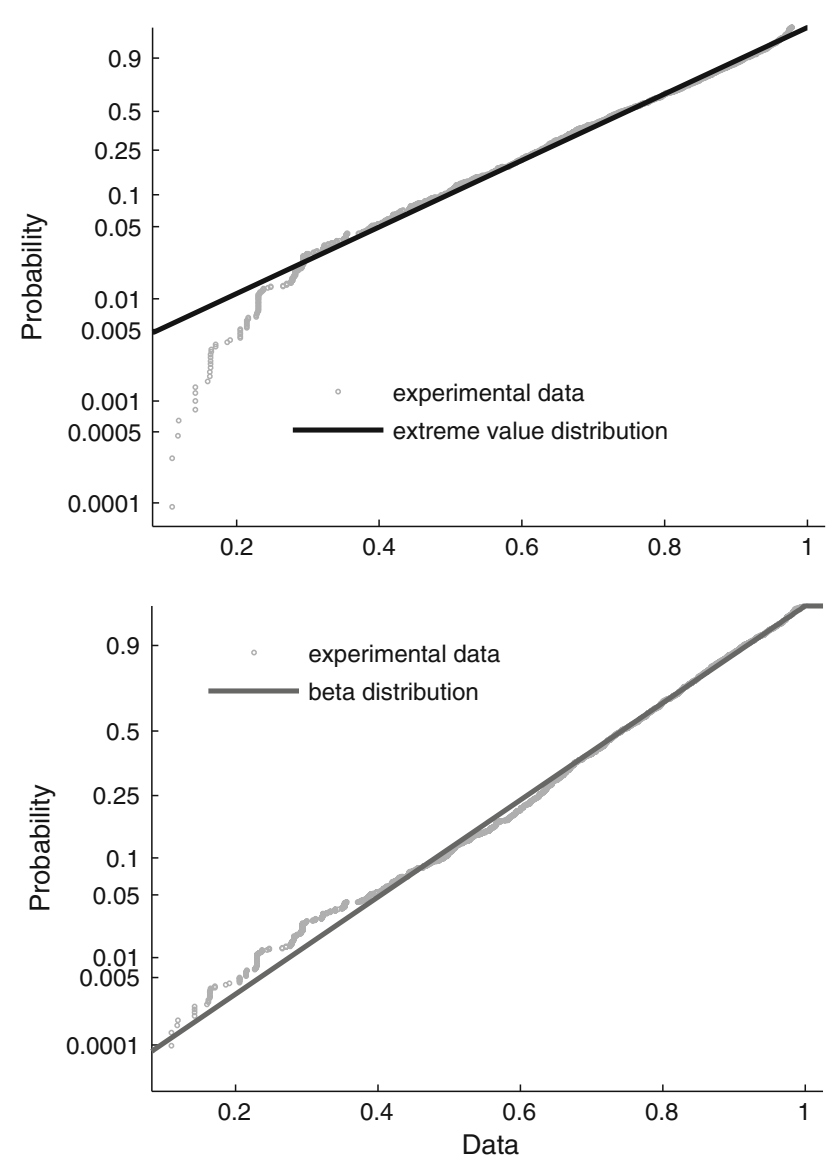

Fig. 8 Probability plot of beta and extreme value distributions versus the experimental data. The experimental data seem to follow better the beta distribution

that the experimental data follow better the beta distribution than the extreme value distribution.

Figure 9 shows probability densities of weighted straightness for groups of different global angle ranges and the corresponding fitted beta distribution. More precisely, Fig. 9a shows the probability density of the weighted straightness parameter $P_{S}$ for fibers with global angles between $-89^{\circ}$ and $60^{\circ},-59^{\circ}$ and $-30^{\circ},-29^{\circ}$ and $0^{\circ}, 1^{\circ}$ and $30^{\circ}, 31^{\circ}$ and $60^{\circ}$, and $61^{\circ}$ and $90^{\circ}$. To compare the distribution for the different angle ranges (Fig. 9a) and the one variable distribution (Fig. 7), all the fits have been plotted in Fig. 9b. The thick black line shows the best fit from Fig. 7 (angle independent density distribution).

Table 3 shows the parameters of the beta distribution fits on straightness parameter distributions for all global angle dependant groups and the univariate set of the data (regardless of global angle). The shape parameters $\alpha$ and $\beta$, related to the univariate $P_{S}$ distribution, were found to be $\alpha=4.7$ and $\beta=1.8$. The distributions pertaining to different angle groups were similar in shape; the shape parameters were $\alpha=$ $4.8 \pm 0.5$ and $\beta=1.8 \pm 0.2$ and differed only slightly in $\alpha$.

The orientational density of local angles as well as the fitted PDF have been plotted in polar coordinates in Fig. 10 for both cut-open and intact groups. For simplicity, the densities are plotted between $-\pi / 2$ and $\pi / 2$. As it can be seen, the experimental data show a four modal distribution. For this reason, we have used a PDF composed of four $\pi$-periodic von Mises distributions, representing four families of fibers. Figure 10a shows the local angle densities related to the cut-open group. Based on the fitted distribution, the four modes occur at $0^{\circ}$ (axial direction), $90^{\circ}$ (circumferential direction), $43^{\circ}$ and $-43^{\circ}$ with circular standard deviation (CSD) of $40^{\circ}, 47^{\circ}, 37^{\circ}$ and $37^{\circ}$, respectively. Based on these derived CSDs, the distribution appears less variable around $43^{\circ}$ and $-43^{\circ}$ than around $0^{\circ}$ and $90^{\circ}$.

Figure 10b shows the local angle distributions for the uncut group. Compared to Fig. 10a (cut group), the distribution is shifted to the circumferential direction. The families of fibers with mean orientations of $43^{\circ}$ and $-43^{\circ}$ have been relocated to mean orientations of $47^{\circ}$ and $-47^{\circ}$, respectively. Furthermore, the value of probability density function has decreased in the axial direction (at $0^{\circ}$ ) by $32 \%$ while it has increased in the circumferential direction (at $90^{\circ}$ ) by $19 \%$.

Figure 11, shows the measured density distribution of local angles for both cut and uncut groups. Figure 11 also shows the predicted density distribution for the intact group based on the distribution of the cut-open group and applying the deformation with $\lambda_{\theta} / \lambda_{z}=1.24$ using Eq. 19. This corresponds to the deformation needed to bring the cut-open group geometry to the intact (uncut) geometry. The results showed that a transformation of the probability density distribution for cut-open arteries using a deformation with equivalent circumferential to axial stretch ratios of $\lambda_{\theta} / \lambda_{z}=1.24$, results in the same distribution as the uncut arteries.

\section{Discussion}

We quantified the waviness and angular distribution of collagen fibers in the adventitia of fresh rabbit common carotid arteries. Our results showed that the straightness of fibers 

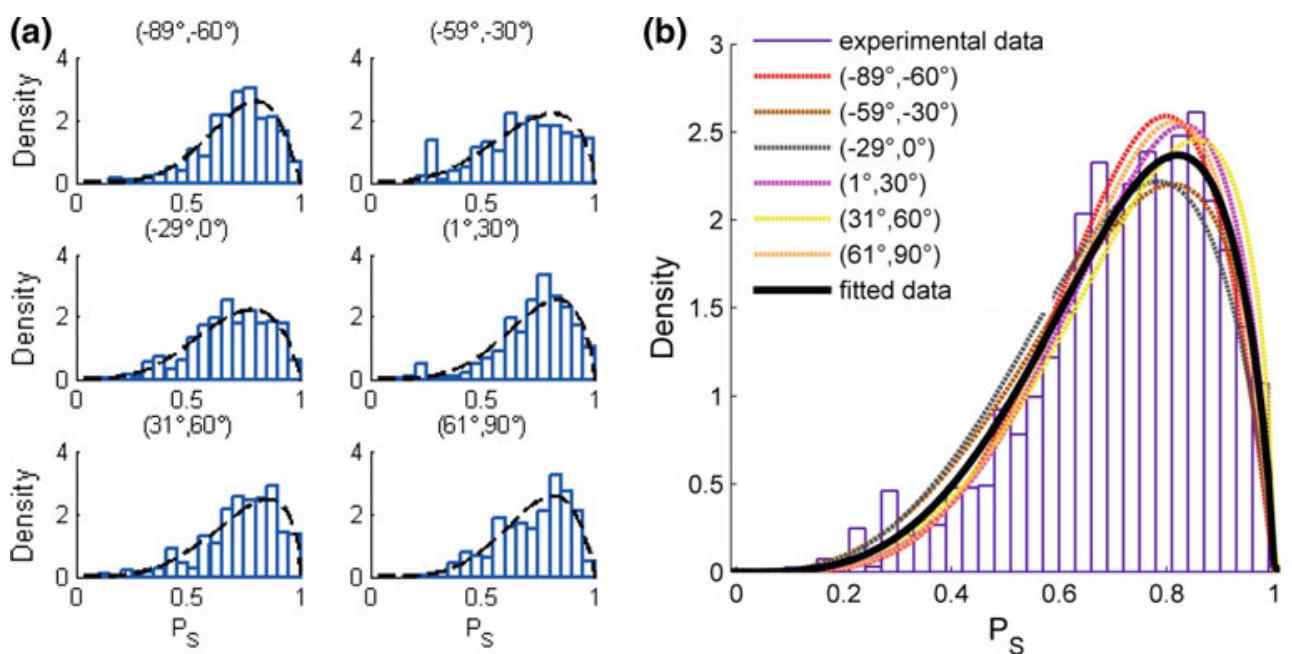

Fig. 9 Distribution of straightness parameter in various ranges of global angles i.e., a beta distribution fitted (dashed line) to experimental data (bars) and $\mathbf{b}$ comparison of all fitted distributions for the different angle ranges (dashed lines) and the angle independent beta distribution (black line)

Table 3 Shape parameters of the fitted beta distributions

\begin{tabular}{llllll}
\hline Angle range & \multicolumn{2}{c}{ Parameter estimation } & & \multicolumn{2}{c}{ Mean and variance } \\
\cline { 2 - 3 } \cline { 5 - 6 } & $\alpha$ & $\beta$ & & Mean & Variance \\
\hline$\left(-89^{\circ},-60^{\circ}\right)$ & 5.5 & 2.1 & & 0.721 & 0.023 \\
$\left(-59^{\circ},-30^{\circ}\right)$ & 4.4 & 1.7 & 0.701 & 0.031 \\
$\left(-29^{\circ}, 0^{\circ}\right)$ & 4.2 & 1.9 & & 0.691 & 0.030 \\
$\left(1^{\circ}, 30^{\circ}\right)$ & 4.9 & 1.8 & & 0.734 & 0.025 \\
$\left(31^{\circ}, 60^{\circ}\right)$ & 4.4 & 1.6 & & 0.736 & 0.028 \\
$\left(61^{\circ}, 90^{\circ}\right)$ & 5.2 & 2.0 & & 0.727 & 0.024 \\
Univariate & 4.7 & 1.8 & & 0.718 & 0.028 \\
set of data & & & & & \\
\hline
\end{tabular}

at the zero-stress state was best represented by a uni-modal beta distribution function with shape parameters $\alpha$ and $\beta$ equal to 4.5 and 1.8. These parameters determine the shape of the distribution and indicate specifically the probability density of the straightness parameter. In addition, the waviness distribution of fibers did not depend on the global fiber angle. The beta distribution fitted the straightness densities for all different groups of global angles, though the values for shape parameters were slightly different in each group. Finally, the automatic analysis of local angle revealed the existence of four main families of fibers. The distribution of angles was fitted by a four modal orientational distribution composed of four $\pi$-periodic von Mises distributions.
Fig. 10 Orientational density of local angles and fitted PDF for cut-open (top) and intact (bottom) groups, in polar coordinates. The four modes with the corresponding CSD are presented on the table

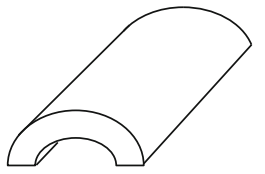

(a) cut-open group

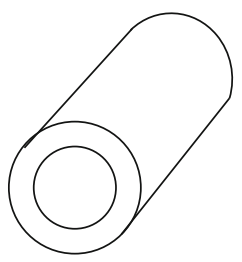

(b) Intact group

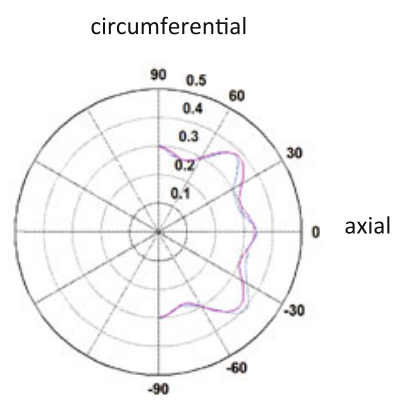

\begin{tabular}{|c|c|c|c|c|}
\hline \multirow{2}{*}{} & \multicolumn{2}{|l|}{ mean(deg) } & \multicolumn{2}{l|}{ CSD(deg) } \\
\cline { 2 - 4 } & cut & uncut & cut & uncut \\
\hline 1st & $\mathbf{0}$ & 0 & $\mathbf{4 0}$ & 53 \\
2nd & $\mathbf{9 0}$ & 90 & $\mathbf{4 7}$ & 38 \\
3 3rd & $\mathbf{4 3}$ & 47 & $\mathbf{3 7}$ & 36 \\
4th & $\mathbf{- 4 3}$ & -47 & $\mathbf{3 7}$ & 36 \\
\hline
\end{tabular}

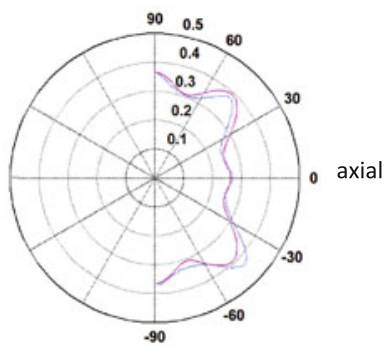

circumferential 


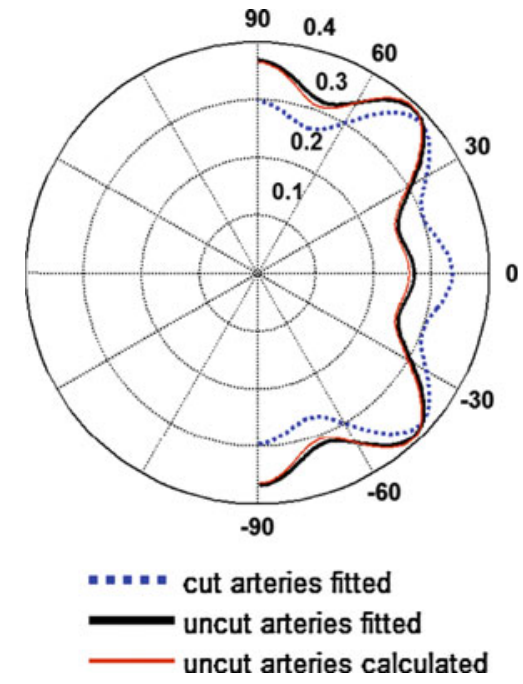

Fig. 11 Measured density distribution of local angles for both cut-open (dotted line) and intact (line) groups, and predicted density distribution of the intact group based on the distribution of the cut-open group. The mapping of local angles of arteries from cut-open to intact state is obtained by using circumferential to axial stretch ratio $\left(\lambda_{\theta} / \lambda_{z}\right)$ equal to 1.24

The local angle distribution of the intact group (uncut arteries) was shifted to the circumferential direction compared to the corresponding distribution of the cut group (zero-stress state). The mapping of the distributions from the ZSS (cut) to the intact group (uncut) could be explained by a simple geometrical transformation, the "folding and joining together", of the cut-open artery (ZSS) to the intact zero-load state, which yields a circumferential to axial stretch ratio of around 1.24 .

\subsection{Collagen visualization}

To visualize collagen fibers in the adventitia of unfixed arteries, we labeled adventitial collagen with CNA35-OG488 fluorescent marker recently developed by Krahn et al. (2006) and imaged the adventitia layer using laser confocal microscopy. The CNA35 probe is specific for collagen present in the cardiovascular tissue (type I and III) and can reveal small collagen fibers as well as more mature structures in living tissues without altering the structure. Therefore, the probe can be potentially used to study collagen remodeling in living tissues (Krahn et al. 2006), e.g., for tissue engineering purposes. Moreover, the probe could be used to visualize collagen fibers of blood vessels under various mechanical loading. The probe has a low binding affinity and does not affect mechanical properties of the tissue, is small, and can thus readily diffuse into the tissue. Collagen fibers and bundles can also be visualized in tissue without labeling agents or fixative as a result of its intrinsic properties such as bifringence under polarized light microscopy (Finlay et al. 1995; Hilbert et al. 1996), auto-fluorescence (Voytik-Harbin et al. 2001), and second harmonic generation (SHG) (Campagnola and Loew 2003; Boulesteix et al. 2006). Techniques for 3D visualization of collagen without specific probes include auto-fluorescence, confocal reflection microscopy (Roeder et al. 2002), and second harmonic generation using femtosecond pulsed infrared excitation (Boulesteix et al. 2006). However, these techniques have some drawbacks. Auto-fluorescence is not specific enough for collagen, as several tissue constituents possess intrinsic auto-fluorescent properties. In addition, auto-fluorescence intensity of collagen is relatively low. Confocal reflection microscopy is subjected to the same drawbacks. As for the SHG, strong forward scattered SHG does enable the visualization of collagen in tissues; however, depending on tissue properties, forward SHG is not always feasible. In dense and thick tissues, such as large arteries, it is difficult to obtain images with the forward scattered SHG. These tissues require additional techniques to image collagen in backward geometry i.e., in direction opposite to the direction of the excitation laser, which results in a much weaker signal. In a recent study, Boerboom et al. (2007) compared the imaging of collagen with CNA35 probe and backward geometry SHG signal. They observed that the SHG in backward geometry was much weaker than the fluorescent signal of the probe. Similarly, this was observed in our preliminary studies on rabbit common carotid arteries. On the other hand, the probe has some limitations mainly due to the diffusion depth into the tissue. Megens et al. (2007) showed limited labeling of the probe in viable elastic and muscular arteries. In our preliminary study on rabbit common carotid arteries, we also observed limited diffusion of the probe through the external and internal elastic lamellae after incubation overnight. In summary, labeling collagen with the CNA35-OG488 enabled us to visualize collagen fibers in fresh unfixed tissue with depth of measurement $\sim 0.1 \mathrm{~mm}$; the thickness of the adventitia layer is about $0.13 \mathrm{~mm}$. The fluorescent signal was collagen specific and strong enough to image the adventitial fibers well and in good detail. However, the diffusion depth of the probe is limited and probably could not be used in arteries with thick elastin lamellae to access collagen fibers in other layers of arterial wall.

\subsection{Analysis of collagen fiber waviness}

We quantified the collagen fiber straightness parameter, $P_{s}$, in the adventitia and calculated its empirical probability density function, which we fitted with a beta distribution. Straightness parameter, $P_{s}$, defined as the ratio of the distance between endpoints and the arc length of the fibers, is the inverse of the straightening stretch of fibers. The straightening stretch is the stretch to be applied along the fiber to get it 
straightened. Therefore, $P_{S}$ distribution is of particular interest for developing structural models of arterial tissue, which take into account the waviness of fibers (Zulliger et al. 2004; Cacho et al. 2007). These studies are mainly influenced by an earlier framework proposed by Lanir (1983). In this framework, to include waviness of fibers, it is assumed that fibers do not bear load at their wavy state and that there is a straightening stretch along the fiber, $\lambda_{s}$, at which the fiber engages and starts bearing load. $P_{S}$ is assumed to have a statistical distribution, which should be characterized at the zero-stress state of the arteries. Despite the fact that a complete characterization of the statistical distribution of waviness or straightness parameter is needed to realistically model the tissue, the number of experimental studies which have addressed the issue are limited (Elbischger et al. 2005).

To the best of our knowledge, this is the first study which quantifies the waviness distribution of collagen fibers in the adventitia under the zero-stress state of arteries. Based on this work, the $P_{S}$ distribution could be fitted with a beta distribution with a mean value of 0.72 and a variance of 0.028 . Both shape parameters are more than $1(\alpha=4.7, \beta=1.8)$ and therefore the distribution is uni-modal. In this study, different distributions such as lognormal, log-logistic, extreme value, gamma, and beta have been fit to the data (results not shown) and it was evident that the beta and extreme value distribution fit the empirical PDF the best. A more detailed study on probability plots of the data for the beta and extreme value distribution, showed that the data were best fitted by the beta distribution (Fig. 8).

Our results have shown that the shape of the PDF for $P_{s}$ was similar for the fibers of groups with different global angles. This means that the same distribution for straightening stretch could be used for different families of fibers in different orientations, or, stated otherwise, the distribution for straightening stretch is not dependent on fiber angle. Shape parameters differed only slightly between groups. Mean and variance of these distributions are shown in Table 3. The difference between variances could be a result of sampling on the distribution as the number of measurements was fairly small (less than 500 for each group of angles). This information is indeed valuable for constructing structural models of arterial adventitia where both waviness and orientational distributions of collagen fibers are included in the model. Moreover, preliminary results (not presented) suggest that thinner bundles are less wavy.

\subsection{Analysis of local angle distribution}

Based on our results, the distribution of local collagen fiber angles included four peaks (modes), suggesting four main orientations and therefore four families of fibers. This led us to the choice of the distribution given by Eq. 15, which consists of four $\pi$-periodic von Mises distributions summed up and normalized between $-\pi / 2$ and $\pi / 2$. The parameters $\mathrm{b}_{i} i=1,2,3,4$ were free to fit the data. The $\mathrm{b}$ parameter of the von Mises distribution determines its circular standard deviation (CSD), as defined in Eq. 13. However, the standard deviations derived from local angle dataset should not be confused with the circular standard deviation of global angles of fibers. The standard deviation of local angle distribution does not only depend on the standard deviation of global angles, but also depends on local variations of angles due to the fiber waviness. The separation between these two variations is not straightforward and requires segmentation of images into areas with a common mean orientation. Elbischger et al. studied local extraction of crimp parameters of collagen fibers from a single image (Elbischger et al. 2004). The images were taken using light microscopy from sliced fixed paraffin-embedded human iliac arteries. The same image analysis method could be applied on the current data to get more detailed information of the standard deviation of global angle of fibers.

Few experimental studies are available on orientation of fibers in the arterial adventitia. Smith et al. (1981) studied the orientation of collagen in the adventitia of human cerebral arteries. The arteries were fixed under a transmural pressure of $100 \mathrm{mmHg}$. They reported an average circumferential direction with a surface component of longitudinal fibers (Smith et al. 1981). Canham et al. (1989) followed the same approach and reported a circumferential direction of fibers $(C S D=22.3)$ for the adventitia of human coronary arteries fixed at $120 \mathrm{mmHg}$. Canham et al. (1992) found the mean angular deviation of alignment of fibers to be $\pm 30^{\circ}$ for human brain arteries fixed at zero transmural pressure. For arteries fixed at $200 \mathrm{mmHg}$, they reported $\pm 7^{\circ}$ for the inner and $\pm 13^{\circ}$ for the mid-adventitia layer, respectively. In addition, in two sections of frozen arteries fixed at $120 \mathrm{mmHg}$, the results showed that the alignment was within the range of measurements for wax embedded tissue. In a recent study, Finlay et al. (1995) reported a mean helical angle of $70^{\circ}(\mathrm{CSD}=36), 53^{\circ}\left(\mathrm{CSD}=32^{\circ}\right)$ and $14^{\circ}\left(\mathrm{CSD}=23^{\circ}\right)$ with respect to the circumferential direction for human brain arteries fixed at 30,120, and $200 \mathrm{mmHg}$. These studies have been mainly performed on sections from fixed and paraffin embedded arteries at a specific luminal pressure. The fixation, dehydration, and paraffin embedding causes shrinkage and could change the morphology of the tissue (Dobrin 1996), albeit a mainly isotropic shrinkage would affect less the orientation data. Moreover, the measurements focus on pressurized blood vessels and do not quantify the organization of fibers at the zerostress state of arteries, as needed for modeling purposes. In addition, none of the studies reported the longitudinal stretch at which the arteries were fixed. In the current study, we have measured the structure-related parameters on fresh 
unfixed tissue and at zero-stress state having in mind that such information is needed to develop structural models of the arterial wall.

In addition to the cut-open arteries, we also imaged the collagen on the adventitia of intact arteries at their zero-load state (ZLS), i.e., at zero transmural pressure and no axial load. The local angle distribution in intact arteries showed a shift to the circumferential direction as seen from Fig. 10. The PDF of the orientation angle in the axial direction $\left(0^{\circ}\right)$ was reduced by around $20 \%$; in the circumferential direction it was increased by $23 \%$. The transformation model predicts a value of 1.24 for $\lambda_{\theta} / \lambda_{z}$, when the arterial geometry goes from the zero-stress state (cut-open) to zero-load state (intact). The relative increase in the circumferential stretch is a direct result of the "closing" of the artery from the ZSS to ZLS, which entrains a significant positive circumferential strain on outer (adventitial) layer of arteries. As adventitial fibers are strained more in the circumferential direction, their mean orientation would also shift to the circumferential direction, which is consistent with our results. This could also explain the results of Finlay et al., where, the higher the pressure at which arteries were fixed and imaged, the closer the circumferential direction was to the mean orientation of collagen fibers. This observation also emphasizes that measurements of fiber angles at zero-stress state could give different results from measurements done on intact or pressurized arteries.

\subsection{Limitations}

There are a number of assumptions and limitations in the methods of the present work. We assumed that fibers were mainly located in the circumferential-longitudinal plane, which allowed us to flatten the 3D data to $2 \mathrm{D}$. This was done in order to facilitate the analysis and particularly the semimanual calculation of the waviness; however, this assumption takes out some useful information such as the contribution of out of plane fibers. In addition, using the 2D data does not help recognize any differences in the orientation or waviness of collagen fibers in different layers of adventitia. Because the data are not used in their full power, further image analysis tools should be developed to study appropriately the $3 \mathrm{D}$ set of data. Moreover, semi-manual technique to calculate the $P_{S}$ parameter is time consuming and the size of fibers are approximated to weigh the data. More elaborate image analysis techniques are needed to automatically segment the 3D images and calculate relevant values such as length and size of the fibers.

The method that has been used to obtain the zero-stress state in the present work gives an approximation of this state, as previous studies have shown that a single radial cut does not reveal the true zero-stress state (Greenwald et al. 1997). The method to visualize collagen fibers with the use of the fluorescent marker needs the arteries to be incubated for a long time. Although all measurements were done within $24 \mathrm{~h}$ of excision, the prolonged storage of the arterial segments could affect the tissue organization and the parameters measured. Finally, most of the bundles of fibers were larger than the size of the obtained image and thus the bundles could be traced only along their length constrained by the size of the image.

\section{Conclusions}

We have quantified the waviness and local distribution of collagen fibers in adventitia of fresh unfixed rabbit common carotid arteries. The results from 50 arterial locations, $\sim 100 \mu \mathrm{m}$ depth each, show the existence of four families of fibers in the zero-load stress state of the arteries. The waviness of fibers does not depend on the fiber orientation. The significant shift of local angular distributions to the circumferential direction from cut-open arteries to intact (no load) arteries shows that angular data from arteries at no load conditions as well as loaded and axially stretched arteries could not be used to define the angular orientation of fibers in ZSS. Despite its limitations, the results of this study could be particularly useful for developing structural models of arterial tissue.

Acknowledgments The authors would like to thank Dr. Dimitrios Kontaxakis for helpful discussions on statistical distributions and Dr. Tyler Thacher for proofreading the article.This work was supported by the Swiss National Science Foundation (Grant No. 325230_125445).

Open Access This article is distributed under the terms of the Creative Commons Attribution Noncommercial License which permits any noncommercial use, distribution, and reproduction in any medium, provided the original author(s) and source are credited.

\section{References}

Axer H, Keyserlingk DGV, Prescher A (2001) Collagen fibers in linea alba and rectus sheaths: II. Variability and biomechanical aspects. J Surg Res 96(2):239-245

Bayan C, Levitt JM, Miller E, Kaplan D, Georgakoudi I (2009) Fully automated, quantitative, noninvasive assessment of collagen fiber content and organization in thick collagen gels. J Appl Phys 105(10): $1-11$

Bigun J, Bigun T, Nilsson K (2004) Recognition by symmetry derivatives and the generalized structure tensor. IEEE Trans Pattern Anal Mach Intell 26(12):1590-1605

Billiar KL, Sacks MS (1997) A method to quantify the fiber kinematics of planar tissues under biaxial stretch. J Biomech 30(7):753-756

Boerboom RA, Krahn KN, Megens RT, van Zandvoort MA, Merkx M, Bouten CV (2007) High resolution imaging of collagen organisation and synthesis using a versatile collagen specific probe. J Struct Biol 159(3):392-399

Boulesteix T, Pena AM, Pagès N, Godeau G, Sauviat MP, Beaurepaire E, Schanne-Klein MC (2006) Micrometer scale 
ex vivo multiphoton imaging of unstained arterial wall structure. Cytometry Part A 69(1):20-26

Braga-Vilela AS, Pimentel ER, Marangoni S, Toyama MH, De Campos Vidal B (2008) Extracellular matrix of porcine pericardium: biochemistry and collagen architecture. J Membr Biol 221(1):15-25

Cacho F, Elbischger PJ, Rodriguez JF, Doblare M, Holzapfel GA (2007) A constitutive model for fibrous tissues considering collagen fiber crimp. Int J Nonlinear Mech 42(2):391-402

Campagnola PJ, Loew LM (2003) Second-harmonic imaging microscopy for visualizing biomolecular arrays in cells, tissues and organisms. NatBiotechnol 21(11):1356-1360

Canham PB, Finlay HM, Dixon JG, Boughner DR, Chen A (1989) Measurements from light and polarised light microscopy of human coronary arteries fixed at distending pressure. Cardiovasc Res 23(11):973-982

Canham PB, Talman EA, Finlay HM, Dixon JG (1991) Medial collagen organization in human arteries of the heart and brain by polarized light microscopy. Connect Tissue Res 26(1-2):121-134

Canham PB, Whittaker P, Barwick SE, Schwab ME (1992) Effect of pressure on circumferential order of adventitial collagen in human brain arteries. Can J Physiol Pharmacol 70(2):296-305

Chambers JM, Cleveland WS, Kleiner B, Tukey PA (1983) Graphical methods for data analysis. Wadsworth \& brooks/Cole Publishing Company, Pacific Grove

Dingemans KP, Teeling P, Lagendijk JH, Becker AE (2000) Extracellular matrix of the human aortic media: an ultrastructural histochemical and immunohistochemical study of the adult aortic media. Anat Rec 258(1):1-14

Dobrin PB (1996) Effect of histologic preparation on the crosssectional area of arterial rings. J Surg Res 61(2):413-415

Elbischger PJ, Bischof H, Holzapfel GA, Regitnig P (2005) Computer vision analysis of collagen fiber bundles in the adventitia of human blood vessels. Stud Health Technol Inf 113:97-129

Elbischger PJ, Bischof H, Regitnig P, Holzapfel GA (2004) Automatic analysis of collagen fiber orientation in the outermost layer of human arteries. Pattern Anal Appl 7(3):269-284

Et-Taouil K, Schiavi P, Levy BI, Plante GE (2001) Sodium intake, large artery stiffness, and proteoglycans in the spontaneously hypertensive rat. Hypertension 38(5):1172-1176

Ferdman AG, Yannas IV (1993) Scattering of light from histologic sectionsA: new method for the analysis of connective tissue. J Invest Dermatol 100(5):710-716

Finlay HM, McCyllough L, Canham PB (1995) Three-dimensional collagen organization of human brain arteries at different transmural pressures. J Vasc Res 32(5):301-312

Franchi M, Fini M, Quaranta M, De Pasquale V, Raspanti M, Giavaresi G, Ottani V, Ruggeri A (2007) Crimp morphology in relaxed and stretched rat Achilles tendon. J Anat 210(1):1-7

Greenwald SE, Moore JE Jr, Rachev A, Kane TP, Meister JJ (1997) Experimental investigation of the distribution of residual strains in the artery wall. J Biomech Eng 119(4):438-444

Han HC, Fung YC (1996) Direct measurement of transverse residual strains in aorta. Am J Physiol 270(2 Pt 2):H750-759

Hansen KA, Weiss JA, Barton JK (2002) Recruitment of tendon crimp with applied tensile strain. J Biomech Eng 124(1):72-77

Hilbert SL, Sword LC, Batchelder KF, Barrick MK, Ferrans VJ (1996) Simultaneous assessment of bioprosthetic heart valve biomechanical properties and collagen crimp length. J Biomed Mater Res 31(4):503-509

Jahne B (1993) Spatio-temporal image processing: theory and scientific aplications. Springer, Berlin
Krahn KN, Bouten CV, van Tuijl S, van Zandvoort MA, Merkx M (2006) Fluorescently labeled collagen binding proteins allow specific visualization of collagen in tissues and live cell culture. Anal Biochem 350(2):177-185

Lanir Y (1983) Constitutive equations for fibrous connective tissues. J Biomech 16(1):1-12

Magnusson SP, Qvortrup K, Larsen JO, Rosager S, Hanson P, Aagaard P, Krogsgaard M, Kjaer M (2002) Collagen fibril size and crimp morphology in ruptured and intact Achilles tendons. Matrix Biol 21(4):369-377

Mardia KV, Jupp PE (2000) Directional statistics. Wiley series in probability and statistics. Wiley, Chichester

Megens RT, Reitsma S, Schiffers PH, Hilgers RH, De Mey JG, Slaaf DW, oude Egbrink MG, van Zandvoort MA (2007) Twophoton microscopy of vital murine elastic and muscular arteries. Combined structural and functional imaging with subcellular resolution. J Vasc Res 44(2):87-98

Meijering E, Jacob M, Sarria JC, Steiner P, Hirling H, Unser M (2004) Design and validation of a tool for neurite tracing and analysis in fluorescence microscopy images. Cytometry A 58(2): $167-176$

Rieppo J, Hallikainen J, Jurvelin JS, Kiviranta I, Helminen HJ, Hyttinen MM (2008) Practical considerations in the use of polarized light microscopy in the analysis of the collagen network in articular cartilage. Microsc Res Tech 71(4):279-287

Roeder BA, Kokini K, Sturgis JE, Robinson JP, Voytik-Harbin SL (2002) Tensile mechanical properties of three-dimensional type I collagen extracellular matrices with varied microstructure. J Biomech Eng 124(2):214-222

Sacks MS, Smith DB, Hiester ED (1997) A small angle light scattering device for planar connective tissue microstructural analysis. Ann Biomed Eng 25(4):678-689

Smith JFH, Canham PB, Starkey J (1981) Orientation of collagen in the tunica adventitia of the human cerebral artery measured with polarized light and the universal stage. J Ultrastruct Res 77(2):133-145

Unser M, Aldroubi A, Eden M (1993) B-spline signal processing. Part I. Theory. IEEE Trans Signal Process 41(2):821-833

Voytik-Harbin SL, Rajwa B, Robinson JP (2001) Three-dimensional imaging of extracellular matrix and extracellular matrix-cell interactions. Methods Cell Biol 63:583-597

Whittaker P, Canham PB (1991) Demonstration of quantitative fabric analysis of tendon collagen using two-dimensional polarized light microscopy. Matrix 11(1):56-62

Wicker BK, Hutchens HP, Wu Q, Yeh AT, Humphrey JD (2008) Normal basilar artery structure and biaxial mechanical behaviour. Comput Methods Biomech Biomed Eng 11(5):539-551

Williams C, Liao J, Joyce EM, Wang B, Leach JB, Sacks MS, Wong JY (2009) Altered structural and mechanical properties in decellularized rabbit carotid arteries. Acta Biomater 5(4):993-1005

Wolinsky H, Glagov S (1964) Structural basis for the static mechanical properties of the aortic media. Circ Res 14:400-413

Xia Y, Elder K (2001) Quantification of the graphical details of collagen fibrils in transmission electron micrographs. J Microsc 204(1): $3-16$

Young AA, Legrice IJ, Young MA, Smaill BH (1998) Extended confocal microscopy of myocardial laminae and collagen network. J Microsc 192(2):139-150

Zulliger MA, Fridez P, Stergiopulos N, Hayashi K (2004) A strain energy function for arteries accounting for wall composition and structure. J Biomech 37(7):989-1000 